\title{
Effect of Controllable Distorted Flux on Magnetic Loss inside Laminated Core
}

\author{
Yang Liu ${ }^{1,2}$, Guang Ma ${ }^{1}$, Yana Fan², Tao Liu², Lanrong Liu ${ }^{2}$, Chongyou Jing ${ }^{2}$ \\ ${ }^{1}$ China State Grid Smart Grid Research Institute, Beijing, China \\ ${ }^{2}$ Institute of Power Transmission and Transformation Technology, Baobian Electric Co., Ltd, Baoding, China
}

Email address:

liuyang_white@hotmail.com (Yang Liu), maguang@sgri.sgcc.com.cn (Guang Ma)

\section{To cite this article:}

Yang Liu, Guang Ma, Yana Fan, Tao Liu, Lanrong Liu, Chongyou Jing. Effect of Controllable Distorted Flux on Magnetic Loss inside Laminated Core. International Journal of Energy and Power Engineering. Special Issue: Numerical Analysis, Material Modeling and Validation for Magnetic Losses in Electromagnetic Devices. Vol. 5, No. 1-1, 2016, pp. 42-47. doi: 10.11648/j.ijepe.s.2016050101.16

\begin{abstract}
This paper investigates the magnetic properties of grain oriented electrical steel under the controllable distorted flux conditions based on a product-level core model, and examines the effects of the distorted flux on magnetic loss inside the laminated cores with different excitation conditions, involving different harmonic phase difference, harmonic order and harmonic contents.
\end{abstract}

Keywords: Modeling distorted flux, Magnetic loss, Magnetic property, Transformer core

\section{Introduction}

In a great deal of applications, some transformers are operated under non-ideal conditions. The input voltage of transformer is not always sinusoidal, which results in a distorted flux in the transformer core. Usually the specific total loss of electrical steel is measured under the condition of sinusoidal waveform for the flux density [1-3]. However, the specific total loss values obtained by the standard method are not applicable when estimating the iron loss in a transformer under the distorted flux conditions [4-6], potentially causing a large increase in the iron loss.

Therefore, in order to estimate the iron loss of a transformer, it is very important to accurately measure magnetic properties under the distorted flux condition. Due to the difficulty of establishing the corresponding measurement system, until now, it is hard to find a detailed report of such magnetic properties measurements of a real laminated core.

In this paper, a measurement system for the magnetic properties of grain oriented (GO) silicon steel lamination under distorted flux conditions is presented. The B-H properties and specific total loss of a laminated core model (LCM) with different distorted fluxes are measured, and the effects of harmonic phase difference, harmonic content and harmonic order on magnetic loss are investigated in detail.

\section{Experimental Setup}

The magnetic properties measurements were carried out based on a LCM, with $45^{\circ}$ mitred step-lap joints, made of GO silicon steel B27R095, BAOSTEEL, as shown in Fig.1. The detailed structural dimensions and some individual design parameters of the LCM are shown in Fig.1 and Table 1.

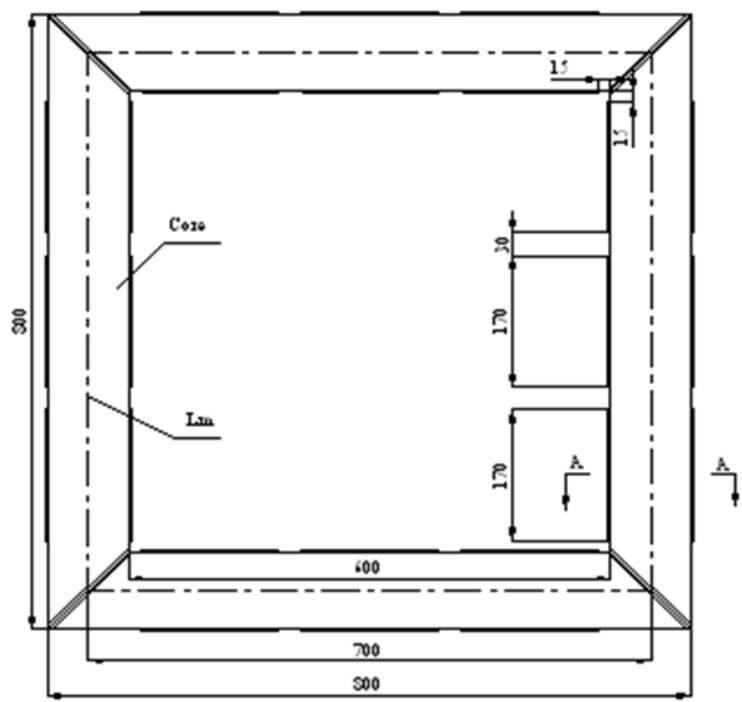

(a) 


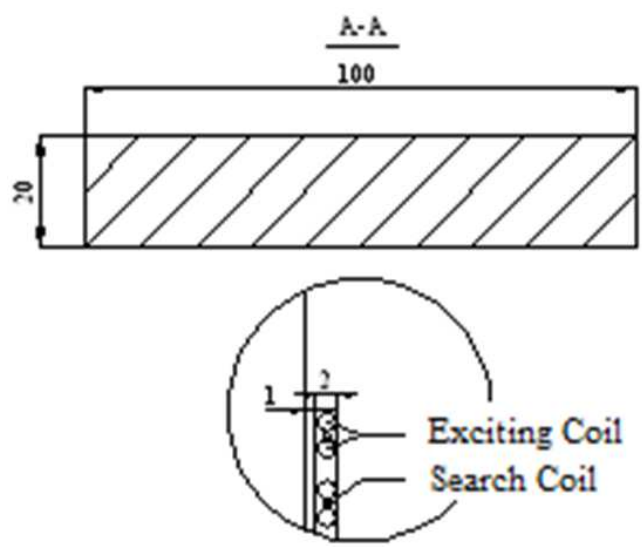

(b)

Figure 1. Laminated core model. (a) structure of laminated core (b) view of cross section.

Table 1. Parameters of core and coils.

\begin{tabular}{ll}
\hline Parameters (LCM) & \\
\hline Number of turns of exciting coil & 288 \\
Number of turns of search coil & 144 \\
Mean length of magnetic path $(\mathrm{m})$ & 2.8 \\
Length of silicon sheet $(\mathrm{mm})$ & 800 \\
Width of silicon sheet $(\mathrm{mm})$ & 100 \\
Thickness of core-leg $(\mathrm{mm})$ & 20.4 (measured) \\
Net area of cross section $\left(\mathrm{mm}^{2}\right)$ & 1978.8 (packing factor: 0.97$)$ \\
Total weight of iron core $(\mathrm{kg})$ & 42.39 \\
\hline
\end{tabular}

The well-established experiment system is shown in Fig. 2. The generator of arbitrary voltage waveforms composed of frequency converter with LC filter is employed as the exciting power source. The waveform and amplitude of flux density in the LCM can be controlled by adjusting the exciting power source.

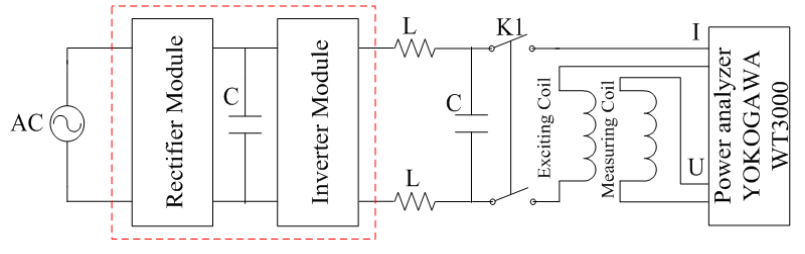

(a)

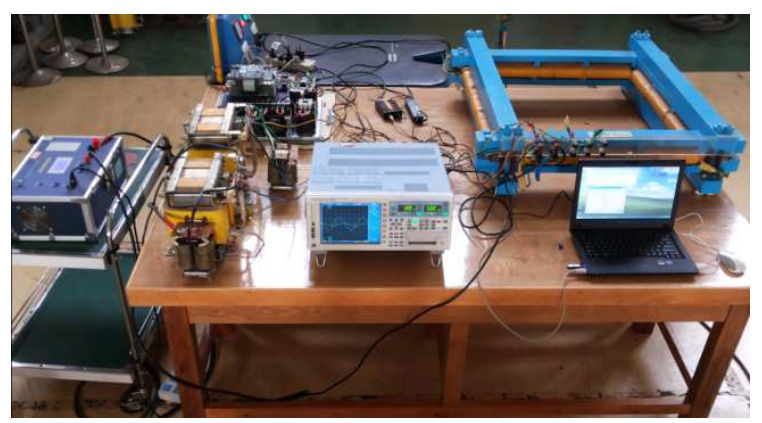

(b)

Figure 2. Experiment system of magnetic property. (a) block diagram of the circuit; (b) experimental apparatus.

\section{Control Method of Flux Density in LCM}

In the measurement, the flux density in LCM can be expressed as follows:

$$
B=\sum_{n=1}^{j} B_{n} \sin \left(n \omega t+\varphi_{n}\right)
$$

where $B_{\mathrm{n}}$ and $\varphi_{n}$ are the peak flux density and phase of the $\mathrm{n}$-th harmonic. The percentage amplitude and the phase difference of the $\mathrm{n}$-th harmonic can be defined as:

$$
k_{n}=B_{n} / B_{1} \times 100 \%, \theta_{n}=\varphi_{n}-\varphi_{1}
$$

where $B_{1}$ and $\varphi_{1}$ correspond to the peak flux density and phase of fundamental content.

In LCM, the induced voltage of exciting coil can be expressed as follows:

$$
E(t)=-N S \frac{d B}{d t}
$$

where $N$ is the number of turns of exciting coil and $S$ is the effective cross area.

Substituting (1) and (2) into (3), the induced voltage of exciting coil can be given as

$$
E(t)=-N S \omega B_{1} \sum_{n=1}^{j} n k_{n} \cos \left(n \omega t+\varphi_{n}\right)
$$

As the resistance and leakage reactance of exciting coil can be neglected in no-load lamination core mode, the exciting voltage can be given as

$$
U(t)=-E(t)=N S \omega B_{1} \sum_{n=1}^{j} n k_{n} \cos \left(n \omega t+\varphi_{n}\right)
$$

In order to realize the control of the flux density in the LCM, the exciting power source is adjusted according to formulation (5).

\section{Measurement Results}

\subsection{The Effects of Harmonic Phase on Magnetic Properties}

The magnetic properties of LCM under distorted flux densities are measured using the above experiment system. It is worthwhile to note that all the distorted flux waveforms presented in the current version of this paper contain only a single harmonic content. Fig. 3 shows the measurement results of B-waveforms and hysteresis loops with the varying phase difference $\theta_{5}$ of the 5 -th harmonic. Fig. 4 shows the corresponding specific total loss of the LCM. The specific total loss at $\theta_{5}=0^{\circ}$ is considerably higher than those from $45^{\circ}$ to $180^{\circ}$, because the larger minor loops occur in the major loop at $\theta_{5}=0^{\circ}$ and the major loop is slightly larger than those from $45^{\circ}$ to $180^{\circ}$ (see Fig.3). Fig.5 and Fig. 6 respectively shows the results of specific total loss at $k_{3}=20 \%$ and $k_{7}=10 \%$. Based on the measured results, it can be seen that the specific total loss shows a decreasing tread with the increase of harmonic phase 
difference at a given harmonic order and harmonic content, $\quad 0^{\circ}$ and $180^{\circ}$.

when the harmonic phase difference varied within the range of

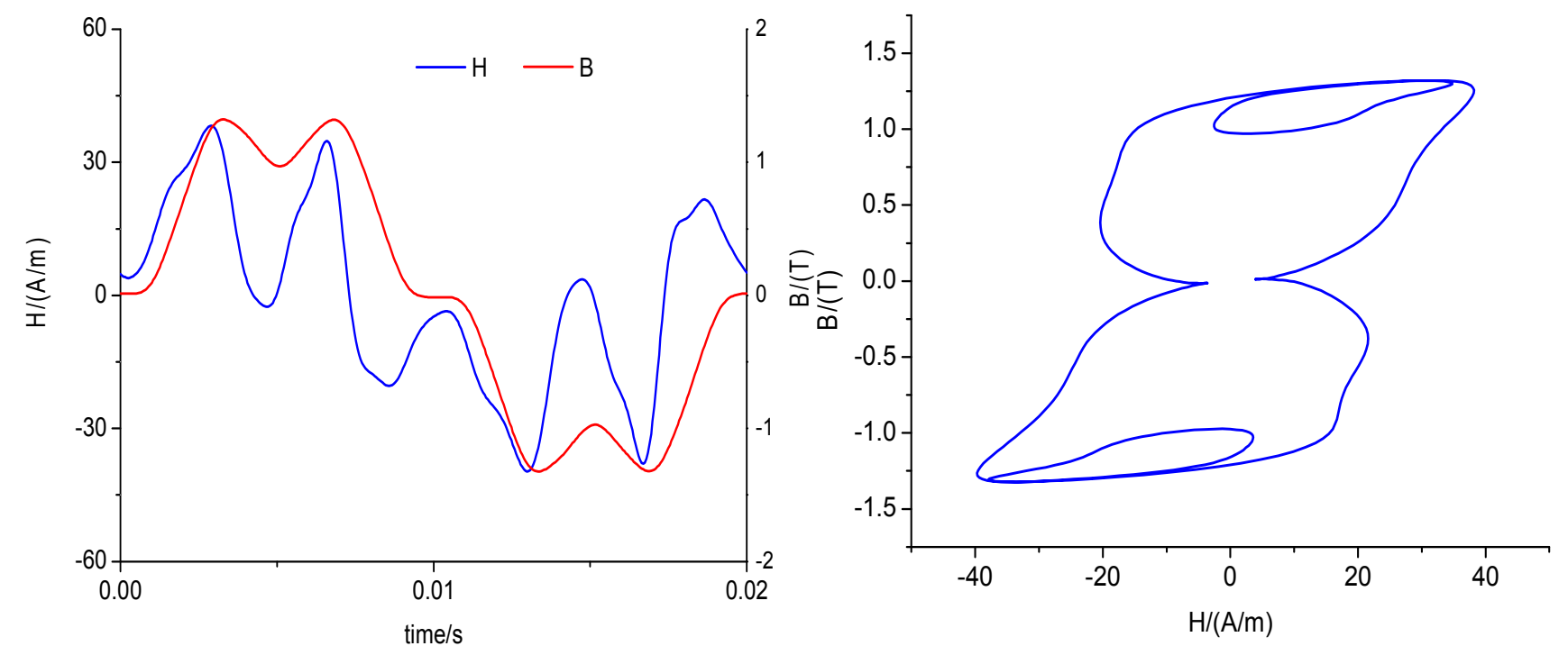

(a)
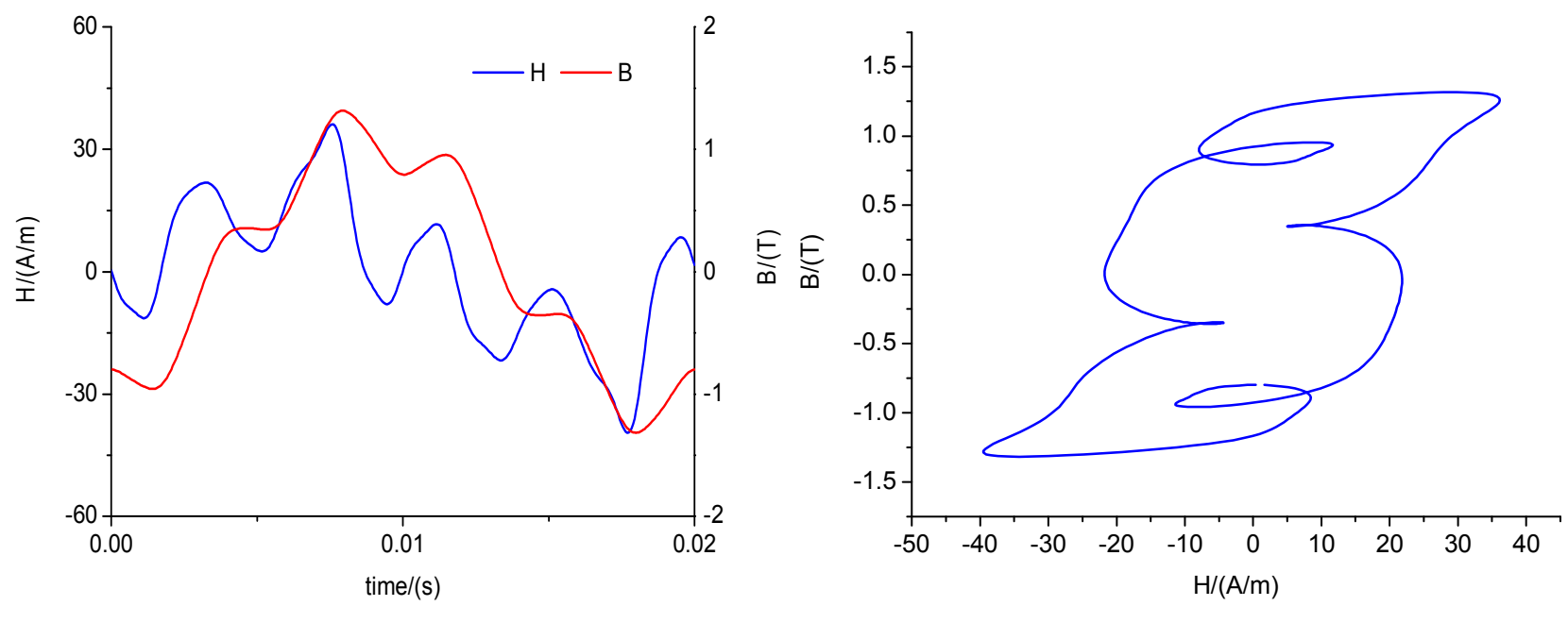

(b)
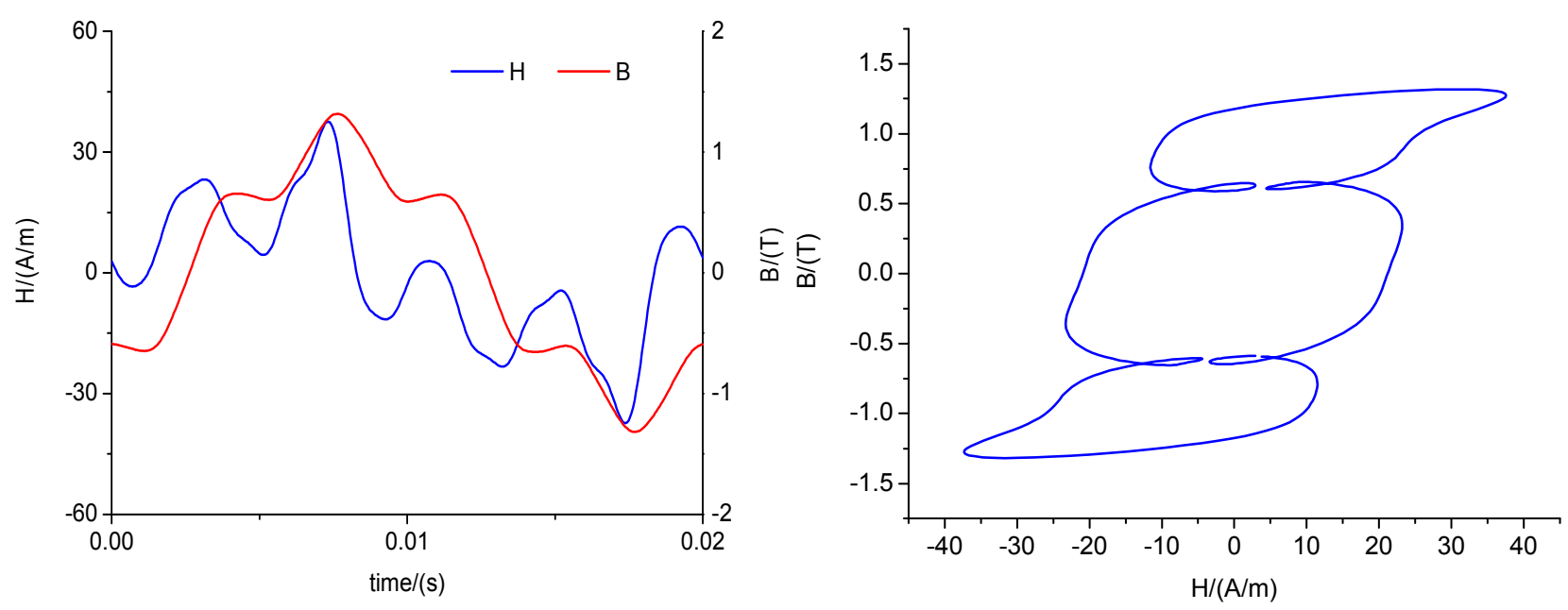

(c)

Figure 3. Measurement results of $B$-waveforms and hysteresis loops at $k_{5}=20 \%, B_{m}=1.32 \mathrm{~T}$ (a) $\theta_{5}=0^{\circ}$ (b) $\theta_{5}=90^{\circ}(\mathrm{c}) \theta_{5}=180^{\circ}$. 


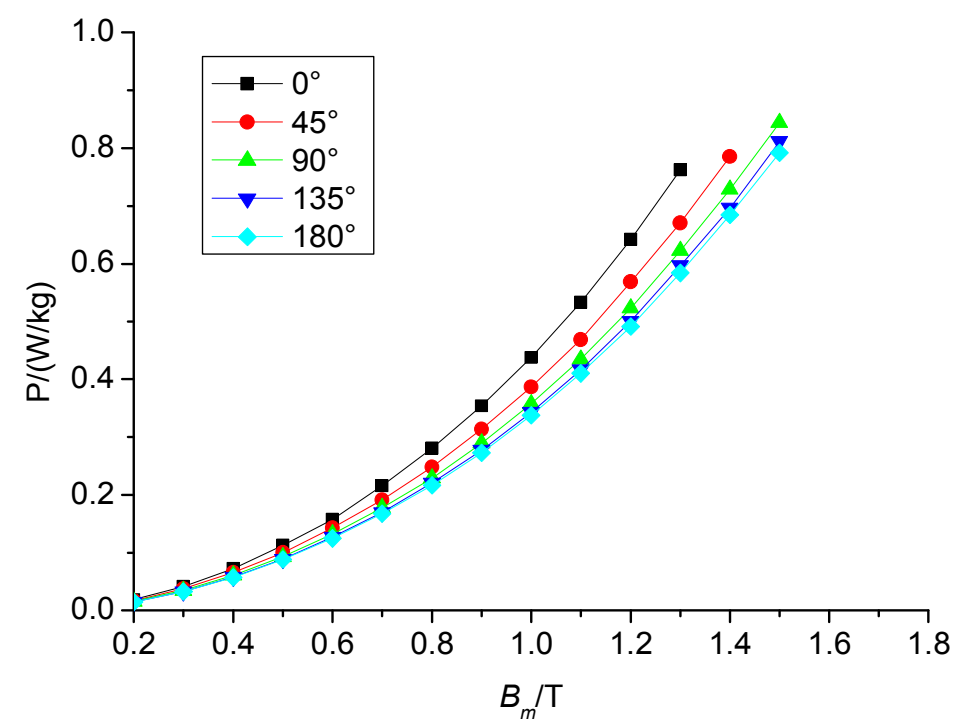

Figure 4. Variation of the specific total loss with the phase difference of 5 -th harmonic $\left(k_{5}=15 \%\right)$.

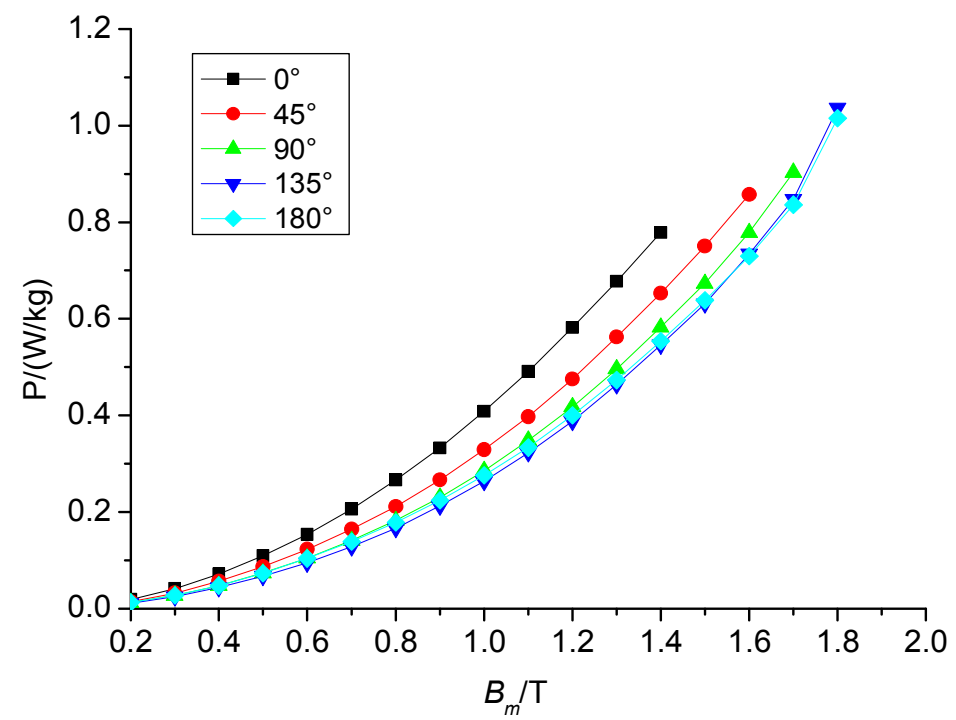

Figure 5. Variation of the specific total loss with the phase difference of 5 -th harmonic $\left(k_{3}=20 \%\right)$.

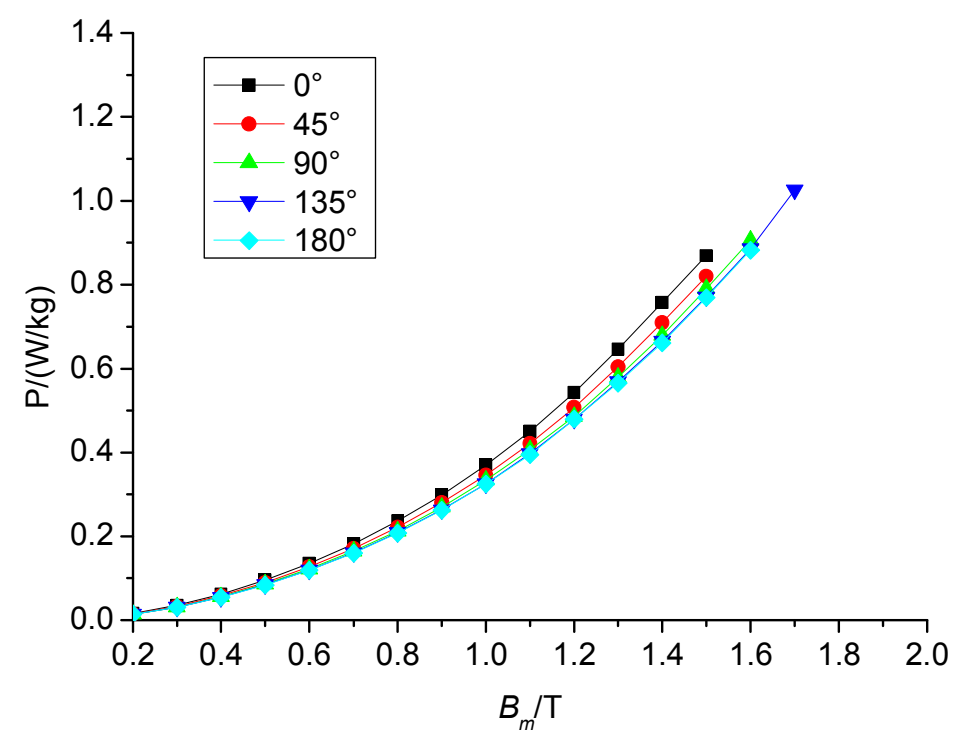

Figure 6. Variation of the specific total loss with the phase difference of 5-th harmonic $\left(k_{7}=10 \%\right)$. 


\subsection{The Effects of Harmonic Content on Magnetic Properties}

The Effects of the harmonic content on the specific total loss are shown in Fig.7 and Fig.8. It can be seen that the specific total loss increase with the variation of the harmonic content, as expected.

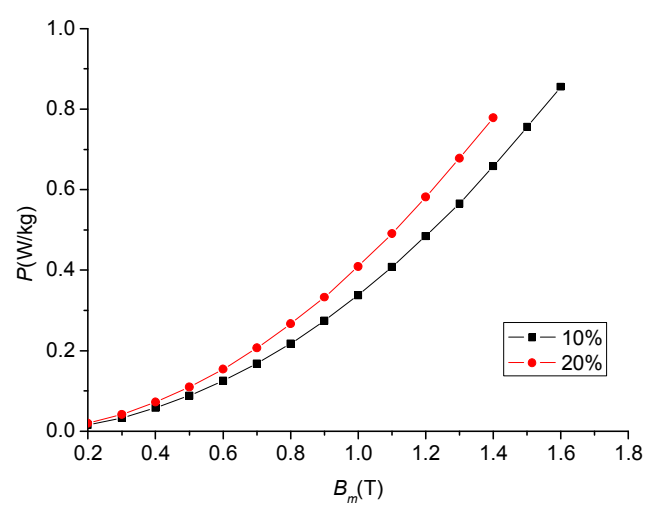

(a)

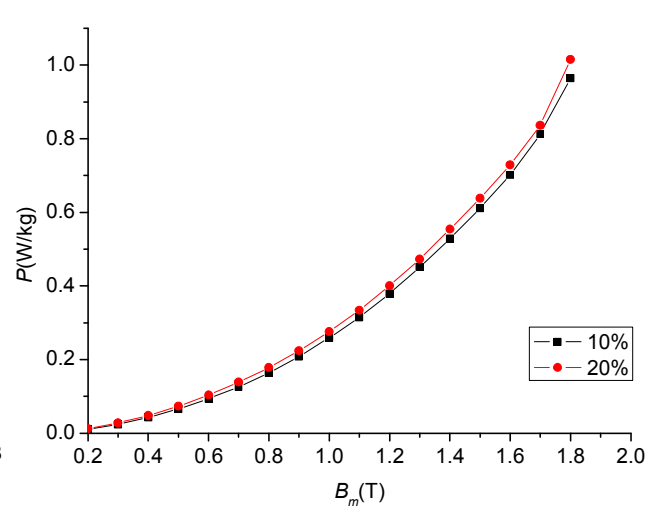

(b)

Figure 7. Variation of the specific total loss with the third harmonic content (a) $0^{\circ}$ (b) $180^{\circ}$.

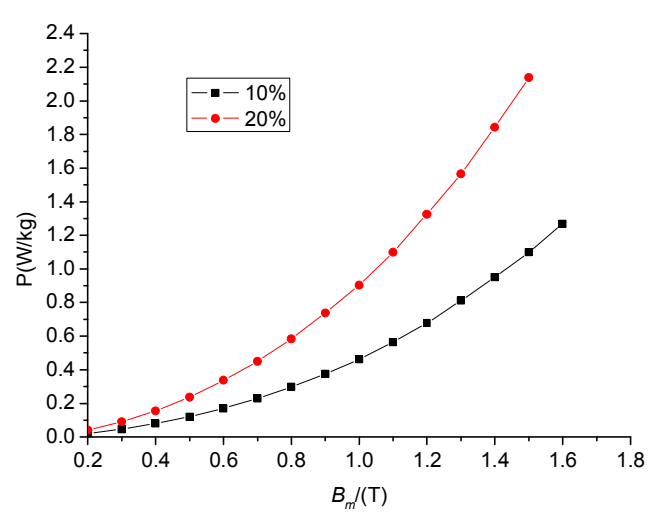

(a)

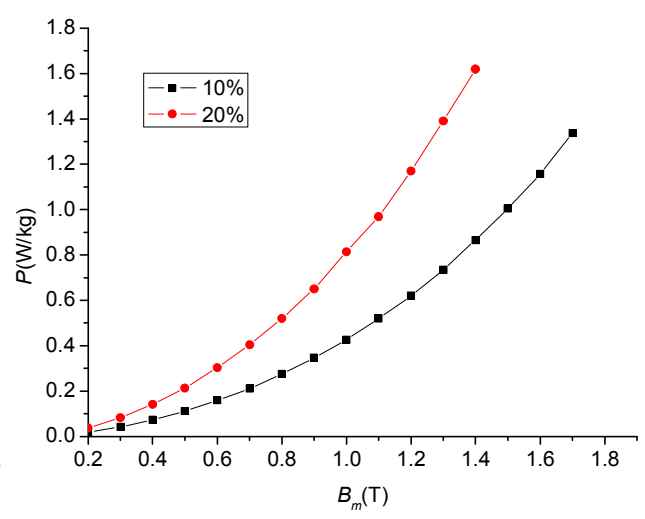

(b)

Figure 8. Variation of the specific total loss with the fifth harmonic content (a) $0^{\circ}$ (b) $180^{\circ}$.

\subsection{The Effects of Harmonic Order on Magnetic Properties}

The variation of the specific total loss with the harmonic orders is shown in Fig.9. The black line with the hollow box is the specific total loss under the sinusoidal flux condition. The specific total loss of $180^{\circ}$ gradually approaches the one of $0^{\circ}$ with the increment of the harmonic order at the same harmonic content. From this result, we can conclude that the specific total loss is likely to be less affected by the phase difference of harmonic when the flux waveforms include the higher harmonic order, which is more obviously affected by the harmonic content and harmonic order.

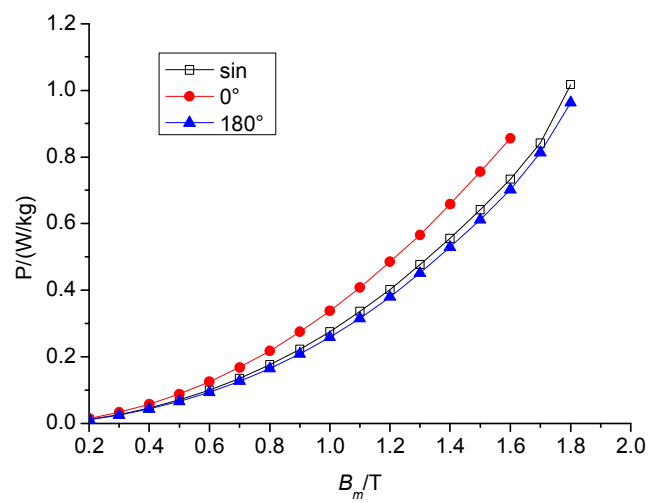

(a)

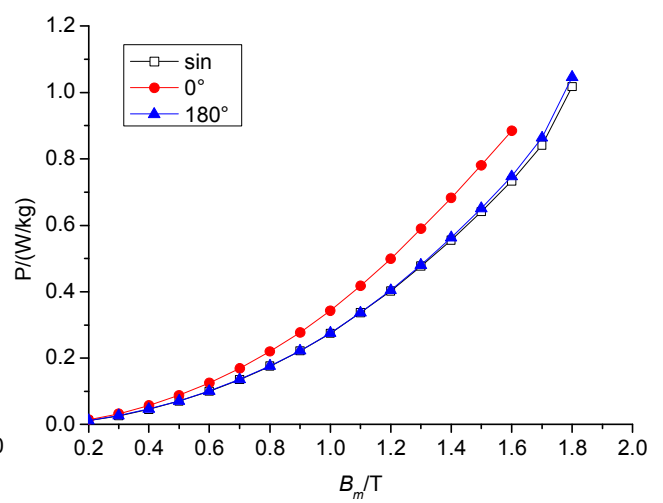

(b) 


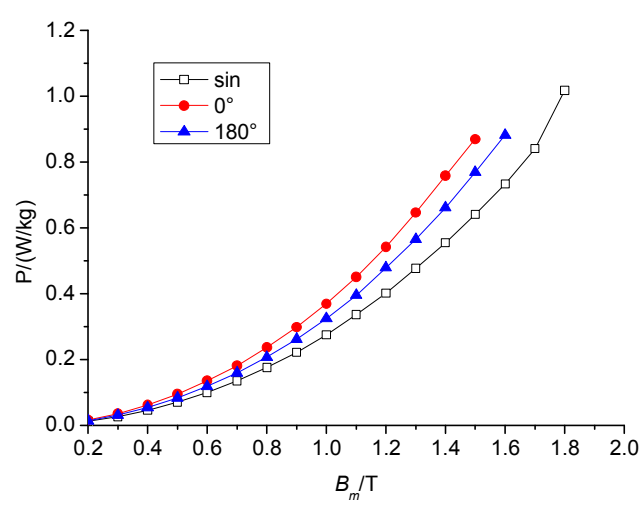

(c)

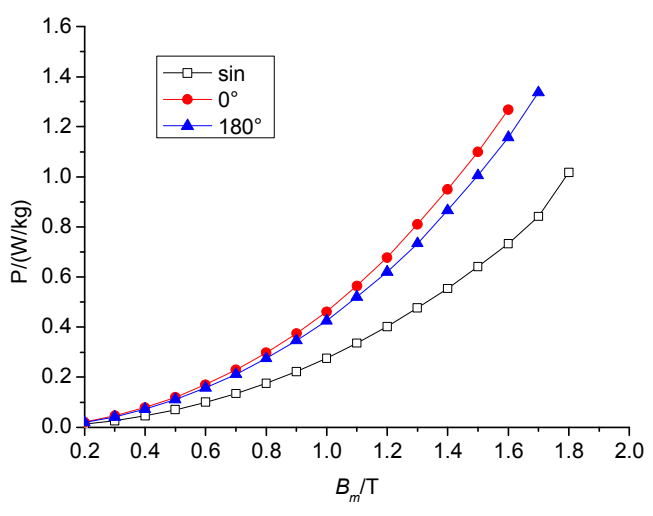

(d)

Figure 9. Variation of the specific total loss with the harmonic order at 10\% harmonic content. (a) the 3-th harmonic (b) the 5-th harmonic (c) the 7-th harmonic (d) the 9-th harmonic

\section{Summary}

A measurement system of controllable flux density for the magnetic properties of GO silicon steel lamination is well established. The effects of harmonic phase difference, harmonic order and harmonic content on iron loss are examined, which can be briefly summarized as follows:

1) The specific total loss shows a decreasing tread with the increase of harmonic phase difference at a given harmonic order and harmonic content, when the harmonic phase difference varied within the range of $0^{\circ}$ and $180^{\circ}$.

2) The effect of harmonic phase difference on specific total loss gradually decreases with the increasing of harmonic order. And the specific total loss is likely to be less affected by the phase difference of harmonic when the flux waveforms include the higher harmonic order.

3) The specific total loss also shows an increasing tread with the increase of harmonic order and harmonic content, as expected.

\section{Acknowledgement}

This project was supported in part by the State Grid Corporation of China under Grants sgri-wd-71-13-002, sgri-wd-71-14-002, and sgri-wd-71-14-009, and by the Youth Science Fund of Hebei Education Department, China, under Grant No. QN20131025.

\section{References}

[1] IEC 60404-2 AMD 1-2008: Magnetic Materials - Part 2: Methods of measurement of the magnetic properties of electrical steel sheet and strip by means of an Epstein frame; Amendment 1.

[2] IEC 60404-3-2010: Magnetic Materials - Part 3: Methods of measurement of the magnetic properties of electrical steel strip and sheet by means of a single sheet tester - Edition 2.2; Consolidated Reprint.

[3] Z. Cheng, N. Takahashi, B. Forghani, et al, "Effect of variation of B-H properties on loss and flux inside silicon steel lamination," IEEE Trans. Magn., vol.47, no.5, pp. 1346-1349, 2011.

[4] A. J. Moses, "Characterisation and performance of electrical steels for power transformers operating under extremes of magnetization conditions," International Colloquium Transformer Research and Asset Management, Croatia, Nov. 12-14, 2009.

[5] A. J. Moses and G. H. Shirkoohi, "Iron loss in non-oriented electrical steels under distorted flux condition," IEEE Trans. Magn., vol. 23, no.5, pp. 3217-3220, 1987.

[6] J. D. Lavers, P. P. Biringer, and H. Hollitscher. "The effect of third harmonic flux distortion on the core losses in thin magnetic steel laminations," IEEE Trans. Magn., vol. PAS-96, no.6, pp.1856-1862, 1977. 